\title{
ALGUNAS REFLEXIONES EN TORNO A LA DIDÁCTICA DE LAS MATEMÁTICAS Y SU ENSEÑANZA
}

\author{
MUMBRÚ, $\mathbf{P}$. \\ Departament de Matemàtica Aplicada i Anàlísi. Universitat de Barcelona.
}

\section{SUMMARY}

This paper raises some basic questions concerning the weak characterization of Mathematical Education as a field of Knowledge and the corresponding effects on the Initial Training of Teachers of Mathematics.

\section{INTRODUCCIÓN}

La didáctica de las matemáticas es, en la actualidad, un ámbito de conocimiento con una definición imprecisa y sin un espacio claramente definido en el panorama científico. Pero los profesores de didáctica de las matemát ${ }^{-}$cas formamos parte de un colectivo encargado de la formación matemática inicial de los futuros educadores. Esto nos sitúa en un contexto nada fácil: hemos de enseñar un conocimiento que no está consolidado y que se encuentra en una fase evolutiva inicial. Aparece pues un conjunto de cuestiones que debemos afrontar: ¿Cómo ha đe ser esta enseñanza? ¿En qué debe basarse?, ¿qué debe pretender?...

Este escrito aborda la problemática que se acaba de exponer, con la intención de proponer ciertas reflexiones en torno a ella que, sin pretender ofrecer respuestas definitivas, nos permitan cuestionar algunas de nuestras concepciones sobre la didáctica de las matemáticas y su enseñanza.

\section{LAS DEMANDAS INICIALES}

¿Cuáles son las expectativas de los alumnos al iniciar un curso de didáctica de las matemáticas? Podemos utilizar sus propias palabras para expresarlas: « ¿Cómo me gustaría poder decir que sé cómo he de explicar las ecuaciones, las raíces cuadradas, la geometría, etc., de manera que mis alumnos lo entiendan!» Este deseo se corresponde con una concepción de la didáctica que considera posi- ble la existencia de unos recursos y unos métodos que son los adecuados para conseguir con éxito las finalidades educativas propuestas y que los alumnos esperan que les sean ofrecidos.

Pero, ¿podemos esperar que la didáctica de las matemáticas dé satisfacción, actual o potencialmente, a estas demandas? La respuesta que demos a esta cuestión estará fuertemente vinculada a la concepción que tengamos de la naturaleza del conocimiento didáctico. De acuerdo con Brousseau (1990-91), podemos tomar en consideración tres acepciones principales ${ }^{1}$ del térrmino didáctica que de manera concisa podemos denominar: técnica, empírico-científica y sistémica. Vamos a precisar cuáles son los puntos de vista esenciales en cada caso y a considerar sus limitaciones.

\section{CONCEPCIONES SOBRE LA DIDÁCTICA}

La concepción «técnica» considera la didáctica como conjunto de técnicas que sirven para enseñar. Por lo tanto, se esfuerza en buscar los mejores métodos de presentar en clase los contenidos matemáticos, de manera que se consigan unos buenos resultados de aprendizaje por parte de los alumnos. Se interesa sobre todo por descubrir nuevos temas para trabajar, nuevos materiales o nuevos puntos de vista a partir de los cuales presentar temáticas conocidas, considerando de manera implícita que, si hasta el momento no se han conseguido mejores resultados, esto es debido a que todavía no se ha encon- 
trado «la buena» manera de hacerlo y de aquí se desprende su interés en buscarla.

El punto de vista «empírico-científico» considera la didáctica como el estudio de la enseñanza en el marco de una disciplina científica. Por lo tanto, planifica situaciones de tipo experimental y las analiza detalladamente, con la pretensión de obtener unos resultados rigurosos y sólidos que permitan aumentar el conocimiento descriptivo, causal y predictivo. Usualmente las reglas y los criterios de legitimidad se toman de disciplinas externas a la didáctica y, muchas veces, el nivel de rigor se consigue mediante la utilización de instrumentos de tipo estadístico que, de este modo, se convierten en la garantía de la fiabilidad de los resultados obtenidos.

La concepción que hemos denominado «sistémica» considera que la didáctica de las matemáticas ha de constituir una ciencia (usando las mismas palabras de Brousseau: una epistemología experimental) que teorice la producción y la comunicación del saber matemático, a la vez que pone un énfasis especial en la necesidad de que sea un ámbito de conocimiento autónomo de otras ciencias y concentrado en aquello de específico del saber matemático que tengan los fenómenos de comunicación y transformación de un saber. Se esfuerza, pues, en producir elaboraciones teóricas, basadas en observaciones experimentales, que lleven a consolidar un cuerpo de conocimientos propios. Aún más, como un resultado de esta consolidación, se aspira a poder fundamentar la profesionalidad de los enseñantes de matemáticas; es decir, a poder disponer de un conocimiento comunicable, con conceptos unificados y con criterios para valorar y analizar las intervenciones y los problemas didácticos, así como precisar las condiciones de uso y reproducción de técnicas y situaciones.

Entre todas estas acepciones se abre a menudo un abismo de incomprensión y de incomunicación que hace difícil el diálogo entre ellas. Pero, si queremos avanzar en la consolidación de una didáctica de las matemáticas como ámbito del saber, resulta indispensable el esfuerzo por aumentar el debate y el acercamiento entre las diferentes posturas implicadas. A mi modo de entender, todas ellas presentan limitaciones y puntos delicados que deben ser tomados en consideración.

En todas las concepciones encontramos un deseo subyacente de mejora del aprendizaje que se expresa mediante una voluntad de innovación y de cambio, siendo la concepción técnica, con su búsqueđa del «buen método», la más explícita en este sentido. Es cierto que los profesores tenemos necesidad de la innovación para sentimos «protagonistas» en nuestra profesión y encontrar nuevos alicientes en el trabajo, que de otro modo resulta excesivamente rutinario y repetitivo. Pero es posible constatar ${ }^{2}$ cómola innovación, entendida como fenómeno colectivo, aporta muy poco al conocimiento didáctico puesto que está sujeta a «los dictados de la moda» y consigue unos productos de escasa duración. Además la innovación pone un énfasis excesivo en la importancia del método, olvidando que existe una trama compleja de infiuencias que condicionan los resultados obtenidos al utilizar un mismo método.
La concepción empírico-científica parte de unas pretensiones de rigor considerables, intentando aproximarse a la didáctica como ciencia. Pero en cambio, existe una gran desproporción entre los medios empleados y las conclusiones obtenidas que, a menudo, están predeterminadas a priori o simplemente ofrecen explicaciones de un nivel primario ${ }^{3}$. Además, en último término, podemos cuestionar la posibilidad de adquirir un conocimiento causal y con capacidad de predicción sobre los fenómenos educativos. Más adelante volveremos sobre este punto.

Si bien el análisis que realiza Brousseau (1990-91), para justificar la necesidad de una didáctica de las matemáticas basada en un conocimiento autónomo, unificado y específico, es contundente y nos puede conducir con facilidad a reconocer su interés, existen algunos puntos en su discurso que creo que metecen una atención especial.

En primer lugar, existe una aspiración general de control sobre los procesos implicados: del maestro sobre las interacciones que se producen en el aula y de la didáctica sobre las técnicas específicas para la enseñanza de nociones y su reproductibilidad; aspectos que nos remiten de nuevo a la cuestión, ya apuntada, sobre la posibilidad de adquirir un conocimiento causal y predictivo sobre los fenómenos educativos.

En segundo lugar, se toma una opción «ideal» particular ${ }^{4}$ sobre el aprendizaje que busca confrontar a los alumnos con unas situaciones de aprendizaje autónomo en las que elaboren sus propios conocimientos. Pero, siempre que el alumno aprende en un sentido real se produce esta elaboración propia (esto puede tomarse como definición de aprendizaje), tanto si hemos querido propiciarlo explícitamente, como si no. En cambio, el hecho de que el alumno llegue a participar personalmente en este proceso no nos dice nada sobre la voluntariedad de dicha acción, ni de cómo se ha llegado a producir. ¿Por qué el alumno debe aprender aquello que nosotros queramos que sepa? Si lo hace por sumisión, ¿qué autonomía esperamos que tenga? ¿Qué pasará cuando rechace el saber que Ie queremos transmitir? ¿Por qué puede llegas a hacerlo?

Por eso, para analizar la conveniencia y la viabilidad de una opción como ésta, no podemos basarnos exclusivamente en criterios sobre los procesos de aprendizaje, sino que deberíamos tener presentes también los procesos inconscientes que condicionan las formas de relación entre profesores y alumnos, así como el papel social de la escuela como institución. Si tenemos en cuenta estos otros factores, ¿qué sentido podemos dar al término «autónomo» como califícativo del aprendizaje?

\section{CAUSALIDAD Y REPRODUCTIBILIDAD EN DIDACTICA}

Volvamos ahora al punto en que nos preguntábamos sobre la posibilidad de adquirir un conocimiento causal y con capacidad de predicción sobre la enseñanza. ¿Podemos concebir la didáctica de las matemáticas como un ámbito de conocimiento que intenta buscar situaciones 
contrastables y generalizables, con capacidad propia para establecer vínculos de causa-efecto y para predecir resultados? $\Varangle \mathrm{O}$ bien esto no es posible $y$, por lo tanto, hemos de abandonar estas pretensiones de causalidad y reproductibilidad?

Existe una concepción muy extendida de la didáctica de las matemáticas que considera que este ámbito de conocimiento no es todavía una ciencia consolidada debido a su juventud. Se argumenta que, de hecho, ésta es una anomalía transitoria, puesto que es una ciencia en proceso de desarrollo, que necesita de la creación de un cuerpo de expertos (mejores y más especializados que los educadores actuales) y de un tiempo histórico suficiente para cambiar su estado actual. Pero la constancia en el desfase entre la práctica educativa y la ciencia correspondiente nos puede hacer pensar que quizás ésta sea una variable esencial y no únicamente un accidente transitorio. ¿No podría ocurrir que una didáctica de las matemáticas con las capacidades apuntadas fuera un proyecto mítico?

Si aceptamos la existencia del «inconsciente» que propone el psicoanálisis, podemos deducir que no existirá una «ciencia» de la educación en el sentido de que sea posible establecer una relación de causalidad entre los medios didácticos empleados y los efectos obtenidos. Tal como dice Millot (1982, pp. 198 y 205 ): «[... I sobre el inconsciente no se manda [...] ninguna teoría pedagógica permite caicular los efectos de los métodos puestos en práctica, porque to que se interpone entre la medida pedagógica y los resultados obtenidos es el inconsciente del pedagogo y el educado [...] Todo lo que el pedagogo puede aprender del análisis y por el análisis es saber poner límites a su acción: saber que no pertenece al orden de ninguna ciencia, sino del arte».

Una posición parecida es la que mantiene el matemático G. Polya (1967), en este caso no mediante argumentos relacionados con el psicoanálisis, sino con planteamientos más personales elaborados a partir de «sus propias reflexiones». En la página 281 del vol. 2, afirma: «La enseñanza no es una ciencia, sino un arte. Esta opinión ha sido expresada tantas veces, por tanta gente, que tengo escrúpulos de repetirla $[\ldots]$ ».

¿Significa esto que no es posible desarrollar rigurosamente la investigación didáctica? Con esta limitación, ¿qué tipo de ciencia puede ser la didáctica de las matemáticas? De acuerdo con las consideraciones anteriores, la didáctica de las matemáticas puede ofrecer elaboraciones teóricas (teorizaciones) que constituyan unas de las ficciones ${ }^{6}$ posibles, ficciones que metaforizen las situaciones didácticas de manera significativa y faciliten su comprensión, aunque no tengan capacidad de control directo sobre ellas. Podríamos resumirlo diciendo que esta investigación puede pretender hacer explícitos los condicionantes de la enseñanza de las matemáticas, pero no debe esperarse que sus aportaciones se transformen en reglas que lleguen a substituir las decisiones que ha de tomar el enseñante en su acción.

De este modo, comprender mejor qué está pasando en el seno de una situación didáctica y ofrecer modelos inter- pretativos del porqué son aspiraciones plausibles. Pero no lo es una aspiración de control rígido sobre ellas. La utilidad de la diđáctica de las matemáticas para la educación reside en la mejora de las relaciones de enseñanza producidas por la influencia de sus interpretaciones sobre la comprensión que tengan los enseñantes de dichas relaciones. En este sentido, la teoría resulta indispensable, pero la influencia apuntada sólo podrá darse si el profesor desarrolla a su vez una labor que nadie más puede hacer en su lugar. Esencialmente una labor de reelaboración retroactiva de sus actuaciones para comprender la particularidad de sus relaciones educativas personales.

\section{RELACIONES EN EL SENO DEL SISTEMA DIDACTICO}

Acabamos de citar una de las limitaciones básicás de la didáctica como ciencia: su incapacidad para producir un control de las relaciones de causa-efecto en las situaciones $y$, por lo tanto, la imposibilidad de decidir por el profesor. Pero hemos de ser conscientes también de otras limitaciones fundamentales de nuestra acción educativa.

Una interpretación inicial de las situaciones didácticas genéricas puede mostrar cuál es el campo reducido en el que los profesores incidimos sobre el aprendizaje de los alumnos. Para hacer explícita la trama de relaciones que existe dentro del sistema didáctico (terna alumnos-matemáticas-enseñante), podemos considerar los siguientes aspectos:

1. Además de las relaciones interpersonales, profesores y alumnos se relacionan con las matemáticas de manera afectiva (ansiedades, inconsciente y deseos), ideológi$\mathrm{Ca}^{7}$ (respecto al conocimiento, al aprendizaje y a las matemáticas) y epistemológica (conocimiento propio de las matemáticas).

2. Estas relaciones se reflejan en unas actitudes didácticas del profesor hacia los alumnos y recíprocamente, condicionando fuertemente el proceso educativo. El profesor delante de los alumnos no está ofreciendo un producto neutro, sino unas matemáticas y unas actuaciones pedagógicas investidas de sus concepciones personales, ocurriendo otro tanto con los alumnos.

3. La actitud del profesor es un factor importante que incide sobre la manera de relacionarse los alumnos (afectiva, ideológica y epistemológicamente) con las matemáticas. La actitud de los alumnos debería incidir también (aunque parece que aquí la situación es asimétrica) en las relaciones de los profesores con las matemáticas, llevándonos a cuestionarlas.

La dinámica de toda esta trama está gobemada por tres principios básicos:

a) Aunque necesite de un contexto social para producirse, el aprendizaje es un proceso personal del alumno; él es quien lo realiza. El profesor no puede «hacer aprender", sino sólo (y no es poco) favorecer o no, con su 
actitud, la posibilidad para el alumno de aprender o no hacerlo.

b) El aprendizaje es un proceso cargado de incertidumbre que comporta cierto grado de frustración y decepción ${ }^{8}$.

c) La eficacia de un método está en función de la comunicación que establezca el profesor a través de él, de aquello que refleje de su actitud.

El aprendizaje depende del alumno en un doble sentido: en la construcción ${ }^{9}$ y en la motivación ${ }^{10}$. Por lo tanto, la influencia del profesor está condicionada en ambos. No obstante, tengamos en cuenta que la capacidad del profesor para «hacer matemáticas», en lugar de ofrecer a los ahumnos respuestas ya elaboradas, hace posible que el alumno interiorice la imagen de una persona que se enfrenta con el proceso de aprendizaje, una persona que piensa y que le muestra que es posible aprender. De este modo es como parece que la influencia del profesor en el aprendizaje del alumno se puede ejercer mejor sobre la construcción del propio conocimiento.

El aprendizaje se produce atravesando por una situación en la que todavía no conocemos cuál es nuestro punto đe llegada, ni hemos conseguido aún lo que nos proponemos. Por eso, particularmente en el caso de las matemáticas, resulta un proceso cargado de incertidumbre, que exige persistencia, que comporta cierto grado de frustración y decepción, y que liega a poner a prueba la autoconfianza en las propias capacidades. Cuando el grado de frustración sobrepasa los límites tolerables para la persona, la actividad de aprendizaje se convierte en una actividad que hay que evitar (renuncia o represión" ${ }^{\text {) }}$ o en una actividad tratada con omnipotencia y rabia (proyección).

Para poder llegar a ser capaces de mantener el interés, a pesar de Ia presión originada en el proceso de aprendizaje, se hace necesaria cierta motivación. El alumno puede encontrar esta motivación tanto a partir de sus propias relaciones con las matemáticas, como a partir de los modelos e influencias externas que reciba. En el primer caso, encontrará la motivación necesaria si ha investido ${ }^{12}$ las matemáticas de manera que sienta que éstas le permiten luchar contra las propias «pulsiones» negativas, participar en la creación de cierta estabilidad personal o realizar una actividad eminentemente creativa y constructiva. En el segundo caso, puede encontrar la motivación a partir de la identificación con las concepciones de otras personas y, en particular, las del profesor.

Esta última vía para estimular Ia motivación de los alumnos resulta ser particularmente delicada para el profesor. Si éste ofrece a sus alumnos unas matemáticas y unas actuaciones pedagógicas investidas de sus concepciones personales, algunos alumnos se sentirán identificados con ellas y esto favorecerá su interés, mientras que otros no lo harán y esto provocará cierta retracción por su parte. Si el enseñante no es consciente de la subjetividad de su representación, no podrá concebir que las matemáticas y las relaciones de enseñanzaaprendizaje sean de otro modo. Ésta le parecerá la única manera objetiva de concebirlas y buscará la manera de imponerla a sus alumnos. Algunos la aceptarán porque coincidirá con la suya, se abrirán al profesor y éste se sentirá tranquilo del éxito que experimentará con ellos. Otros la aceptarán por docilidad, a costa de su autonomía personal y llegarán a ser los alumnos «aplicados». Finalmente existirán los que mantendrán una actitự poco identificada con la del maestro y que correrán el riesgo de acabar con alguna de las etiquetas de «tonto»o «vago ${ }^{13}$.

En el tercer principio nos volvemos a encontrar con la cuestión ya apuntada de la eficacia de los métodos pedagógicos. Para ilustrar el papel preponderante de la actitud del profesor (que es de difícil control) respecto al método en sí mismo, podemos utilizar las aportaciones de dos enfoques bien distintos. Para Mauco (1973), «[...] el valor educativo del profesor no depende tanto de aquello que dice o hace, sino de aquello que siente en profundidad, es decir, de su grado de madurez personal. Desde este punto de vista, la misma intervención educativa puede ser favorable o desfavorable al desarrollo del alumno según cuál sea la carga afectiva subyacente a dicha acción». Por otra parte, Broussean (1990-91) propone lo que llama un posible teorema didáctico: «[...] si los profesores creen suficientemente en la eficacia de un método didáctico hasta el punto de descansar completamente sobre él [...] dejan de cumplir su papel en la negociación del contrato didáctico, y el método fracasa».

\section{UN ÁMBITO DE INTERÉS ESPECÍFICO}

La educación escolar, la podemos considerar como una iniciación al mundo de los adultos, estimulada afectivamente por el modelo del maestro a través de la identificación, que está vinculada con la madurez personal del educador. Por lo tanto, las relaciones maestro-alumnos resultan en general un objeto de estudio psicopedagógico, pero la existencia de un tercer polo en el interior del sistema didáctico (las matemáticas, en nuestro caso) origina un ámbito de interés específico para la didáctica de las matemáticas que trasciende el marco escolar estricto.

Si hasta ahora nos hemos referido a la didáctica de las matemáticas como ciencia teórica, debemos distinguir esta consideración de lo que es la práctica educativa de la formación de profesores de matemáticas. Si nuestra responsabilidad consiste en planificar y llevar a cabo la enseñanza de la didáctica de las matemáticas, de acuerdo con lo expuesto en apartados anteriores, debemos mantener vivo el espíritu de observación y análisis en nuestra actividad profesional y desarrollar un mejor saber sobre nosotros mismos y nuestra práctica. Y en ella hemos de ser coherentes con nuestras propias concepciones sobre la didáctica de las matemáticas, hemos de dejar espacio suficiente para las refiexiones de nuestros alumnos, hemos de ser suficientemente flexibles para que ellos puedan efectuar sus propias elecciones y hemos de ofrecer una visión mínimamente integrada y utilizable de las aportaciones de la investigación didáctica.

Si queremos avanzar en un proceso de este tipo, deberíamos comenzar por cuestionar nuestra actitud educativa, 
intentando incidir sobre las distintas vertientes de la trama de relaciones profesor-matemáticas-alumnos (conocimiento matemático, concepciones sobre las matemáticas y la educación, y relaciones afectivas) y buscando, a su vez, la necesaria reelaboracion retroactiva de nuestras actuaciones.

Con el fin de propiciar un primer intento de afrontar esta problemática y a modo de posible guión de referencia, propongo algunas reflexiones iniciales en torno a los puntos citados.

1) Sobre el conocimiento matemático. Nuestra relación epistemológica con el saber matemático puede llegar a tener un carácter eminentemente pasivo $o$, por el contrario, podemos mantener un interés vivo y especiffico por las matemáticas, trabajando en su reorganización y recontextualización, y seleccionando y analizando diversas maneras de aproximarse o de considerar los contenidos matemáticos objeto de enseñanza, con la intención de aumentar nuestro conocimiento sobre las maneras de relacionarse con el saber matemático y sus procesos de producción y, a su vez, de contribuir al aprendizaje de los alumnos.

2) Sobre la concepción de las matemáticas. Podemos preguntarnos cómo encajan nuestras propias concepciones con algunas hipótesis básicas sobre la actividad matemática, que quizás no sean muy usuales, como las siguientes:

a) Las matemáticas son una actividad humana que forma parte de la cultura y está sometida, por tanto, a influencias internas y externas a ella. En el control de las ideas sobre los objetos matematicos y su evolución, sobresalen el papel de una «intuición cultural» ${ }^{14}$ compartida por la comunidad matemática y el de la falibilidad y la existencia de «errores».

b) La resolución de problemas es la fuente esencial del significado del conocimiento matemático.

c) Existen ciertos procedimientos que conforman el núcleo básico de lo que podemos llamar «la actividad matemática». Por ejemplo, plantearse cuestiones, explorar los contextos asociados a ellas, hacer explícitos ciertos conceptos que estamos utilizando y definirlos, realizar conjeturas, etc.

d) Aunque existe una tradición que clasifict y agrupa el conocimiento matemático que đebe ser enseñado por áreas como aritmética, álgebra, geometría, etc, este conocimiento aicanza su mayor potencialidad cuando es considerado desđe un punto de vista más global y unitario.
3) Sobre las concepciones didácticas. ¿Podemos resituar nuestra relación con el saber didáctico, asumiendo sus limitaciones y valorando su importancia como medio para salir del marco cerrado de nuestra actitud educativa ya formada?

A menudo nuestras concepciones didácticas tienden a estar investidas de una voluntad de control sobre los alumnos y su desarrollo. Tenemos la tentación de satisfacer un deseo de modelar a los alumnos en función de nuestros ideales y esperamos poder conseguirlo mediante ciertas técricas pedagógicas.

Pero, ¿no esconde este deseo una ilusión ${ }^{15}$ de omnipotencia (lo podremos conseguir) y de perfección de nuestros ideales? Los intentos de satisfacer este deseo ¿qué llegarán a comportar para los alumnos? ¿No deberíamos saber poner límites a nuestra acción, dejando espacio para una realidad de discordia entre nuestro bienestar psíquico y nuestros deseos? En suma, ¿cómo podemos asumir la necesidad de la educación y, a su vez, su nocividad? Necesidad que surge para satisfacer una exigencia social basada en el convencimiento de que ciertos conocimientos y ciertas conductas nos ayudan a progresar colectivamente. Nocividad que se desprende de las presiones que la misma sociedad ejerce, para mantenerse como tal, sobre los individuos.

4) Sobre la gestión de las relaciones. ¿Hasta qué punto reconocemos la complejidad de la relación con los alumnos y exploramos la pluralidad de estas relaciones, ta dificultad de su gestion y su incidencia en nuestro comportamiento?

Necesitamos un marco $0^{16}$ para pensar sobre la naturaleza de las relaciones, para verlas desde un punto de vista más objetivo y obtener interpretaciones. Con él, si somos capaces de detenernos a observar, escuchar y pensar, a partir de las reacciones de los alumnos hacia los demás podremos hacernos una idea de sus concepciones.

Aunque las interpretaciones por sí mismas no son suficientes, puesto que debemos actuar y tomar decisiones, sí que pueden ayudarnos a tomar conciencia clara de la incidencia que tienen sobre nuestro comportamiento las expectativas de los alumnos (idealización, dependencia, autoridad punitiva, envidia agresiva...), aun siendo irreales, y de la necesidad de los profesores de sustraerse a su presión y de ofrecer, en cambio, a los alumnos experiencias distintas a las temidas o deseadas irracionalmente, las cuales les brinden oportunidades para reajustar sus concepciones. 


\section{NOTAS}

${ }^{1}$ Brousseau considera tambjén otra acepción que consiste en identificar enseñanza y diđáctica. Comoen este caso la didáctica no resulta ser una elaboración «para» o «sobre» la enseñanza, sino que se confunde con la misma acción, no tomaremos en cuenta esta acepción. En el mismo escrito también se refiere a la investigación-acción; su análisis sobre el origen, motivaciones y deficiencias de dicha concepción resulta suficientemente clara y contundente, y no añadiré ningún comentario a sus consideraciones.

2 Ver Broussean (1990-91).

${ }^{3}$ Kapadia (1977) da un ejemplo extremo de este nivel primario de explicaciones que entra en contradicción con las pretensiones iniciales de rigor. En las pruebas utilizadas por el «Schools Mathematics Study Group" (EEUU) los alumnos que trabajaron con su proyecto obtuvieron peores resultados que los que lo hicieron en un curso tradicional. La explicación que dieron los investigadores del SMSG fue que ésta no era una debilidad de sus alumnos sino de las pruebas que se utilizaron: "hacían referencia a objetivos distintos a los de su programa».

${ }^{4}$ Esta opción está vinculada con lo que llamamos, más adelante, aspectos «ideológicos» de la relación con el saber.

${ }^{5}$ Aquí se plantea la cuestión de la relatividad y la falibilidad de los criterios para evaluar estas teorizaciones. Por ejemplo, la concepción sistémica permite disponer de aportaciones teóricas mediante las cuales podemos evaluar el modelo Dienes, que en sí es otra teorización, y constatar sus aspectos inadecuados (Brousseau 1990-91). Pero, no podemos esperar que este proceso no se repita con otras teorizaciones que tomen como objeto de análísis las aportaciones sistémicas.

${ }^{6}$ Ver Blanchard-Laville y Overtelli (1989) y Mannoni (1979).

\section{REFERENCIAS BIBLIOGRÁFICAS}

BLANCHARD-LAVILLE, C. y OBERTELLI, 1989. Rapport à savoir mathématique et médiation didactique, en J. Beillerot et al.,Savoir etrapportau savoir (Eds. Universitaires: Bégédis).

BLANCHARD-LA VILLE, C., 1989. Questions a la Didactique des Mathématiques, Revue Française de Pédogogie, 89, pp. 63-70.

BOUVIER, A., 1987. Didactique des mathématiques. (Cedic/ Natan: París).

BROUSSEAU, G., 1990. ¿Qué pueden aportar a los enseñantes los diferentes enfoques de la didáctica de las matemáticas?, Enseñanza de las Ciencias, Vol. 8(3), pp. 259-267 y Vol. 9(1), pp. 10-21.

DOLTO, F., 1974.Psicoanálisis y pediatría. (Siglo XXI: Madrid).

KAPADIA, R., 1977. Curriculum developement: Practice and theory, Educational Studies in Mathematics, Vol. 8, pp. 199. 200.
${ }^{7}$ El término ideología se usa aquí entendido como conjunto de conceptos, creencias e ideales utilizados para explicar ciertos fenómenos.

${ }^{8}$ Ver Salzberger-Wittenberg y otros (1989), p. 76.

- Este sentido se refiere a la hipótesis que asegura que «los alumnos construyen su propio conocimiento, su propio significado».

${ }^{10}$ Ver Nimier (1976,pp. 186-187) o Millot (1982). Esta últimá afirma : «De hecho, desde un punto de vista psicoanalítico, los mérodos de transmisión de conocimientos importan poco frente al deseo del alumno de aprender.»

"Ver los «mecanismos de defensa» hacia las matemáticas propuestos por J. Nimier (1988) en el capítulo Ill de su libro.

12 Ver Nimier (1988), pp. 135-139.

${ }^{33}$ Ver la entrađa «Affectivités» de J. Nimier en Bouvier (1987). También Dolto (1974) llamá la atención sobre las dos maneras básicas de justificar las dificultades escolares de un alumno: la anormalidad intelectual y la pereza o mala voluntad; argumentando cómo las dos actitudes, pudiendo ser tan falsa una como otra, tienen como resultado fijar aún más al alumno en el círculo vicioso de sus síntomas.

14 Ver Wilder (1981).

15 Ver Millot (1982), pp. 114-205.

16 Según I. Salzberger, la «transferencia» nos puede ofrecer este marco (Salzberger-Witenberg 1989)
MANNONI, M., 1979. La théorie comme fiction. (Seùl: París).

MAUCO, G., 1973. Psicoanàlisi i educació. (Nova Terra: Barcelona).

MILLOT, C, 1982. Freud antipedagogo. (Paidós: Barcelona).

NIMIER, I., 1976. Mathêmatique et affectivité. (Stock: París)

NIMIER, J., 1988. Les modes des relations aux mathématiques. (Meridiens Klincsieck: París).

POLYA, G,, 1967. La découverte des mathématiques, 2 vols. (Dunod: París).

SALZBERGER-WITTENBERG, I., HENRY, G. y OSBORNE, E., 1989. L'experiencia emocional d'ensenyar iaprendre. (Rosa Sensat-Edicions 62: Barcelona).

WILDER, R.L., 1981. Mathematics as a cultural system. (Pergamon Press: Oxford). 